\title{
Plasmonic linewidth narrowing by encapsulation in a dispersive absorbing material
}

\author{
Ryan Peck, ${ }^{1}$ Ali Khademi $\odot,{ }^{2}$ Juanjuan Ren, ${ }^{3}$ Stephen Hughes $\odot,{ }^{3}$ Alexandre G. Brolo $\odot,{ }^{4}$ and Reuven Gordon $\odot^{2, *}$ \\ ${ }^{1}$ Department of Physics and Astronomy, University of Victoria, Victoria, BC, Canada, V8W $2 Y 2$ \\ ${ }^{2}$ Department of Electrical and Computer Engineering, University of Victoria, Victoria, BC, Canada, V8W $2 Y 2$ \\ ${ }^{3}$ Department of Physics, Engineering Physics and Astronomy, Queen's University, Kingston, Ontario, Canada, K7L $3 N 6$ \\ ${ }^{4}$ Department of Chemistry, University of Victoria, Victoria, BC, Canada, V8W $2 Y 2$
}

(Received 17 April 2020; revised 30 June 2020; accepted 17 December 2020; published 7 January 2021)

\begin{abstract}
It is often assumed that adding losses to a resonant system necessarily broadens the width of the resonance. Here, the scattering spectrum of gold nanoparticles in P3HT (strongly absorbing) and PMMA (low loss) were measured using dark field microscopy and surprisingly, the linewidth was halved in the lossy medium. To gain physical insight into this unusual result, a Green function analysis was employed, and the roles of the permittivity, both magnitude and dispersion, in reducing the linewidth when material losses are increased were made clear. This surprising route to narrower linewidths is intriguing for nanoplasmonic applications including sensing, spectroscopy, imaging and nanolasing and also more generally for resonant systems where loss is conventionally avoided.
\end{abstract}

DOI: 10.1103/PhysRevResearch.3.013014

\section{INTRODUCTION}

Resonances are ubiquitous in physics and through the Kramers-Kronig relations [1,2] always possess loss [3]. A canonical representation of the damped simple harmonic oscillator is embodied by the inductor-capacitor-resistor electrical circuit [3]. It has been shown that plasmonic resonances are simply nanoscale versions of these circuits [4]. The general understanding is that the linewidth increases with the damping [3].

The interplay between the linewidth and damping has been studied extensively in nanoplasmonics, mainly attributing linewidth increases to the metal loss or radiative loss [5]. Some works have also studied the influence of embedding in a surrounding lossy medium, again leading to increased linewidths [6-8]. Yet, looking at a simple constant loss does not capture the dynamics of being embedded in a strongly absorbing material. Here we show that richer physics is present when we consider embedding plasmonic nanoparticles in a strongly absorbing material including dispersive effects.

While the general consensus is that loss increases the linewidth of plasmonic resonances, some have noted the advantages of loss in thermally driven applications like thermophoresis and melting for information storage [9] and reducing spontaneous emission noise in amplifiers [10]. There are many plasmonic applications such as surface plasmon resonance (SPR) sensors, spasers (surface plasmon amplifi-

\footnotetext{
*rgordon@uvic.ca

Published by the American Physical Society under the terms of the Creative Commons Attribution 4.0 International license. Further distribution of this work must maintain attribution to the author(s) and the published article's title, journal citation, and DOI.
}

cation by stimulated emission of radiation), and nonclassical light sources that would benefit from narrow linewidths in the resonance [11-22].

For metal losses, many different damping effects have been considered including radiative damping [23,24], Ohmic damping [25,26], surface scattering [27,28], chemical interface damping [29,30], Landau damping [31,32], and interband transitions $[33,34]$. The relative contribution of each of these effects has been debated (for example, Ref. [35]); however, we are not considering the influence of metal losses in this manuscript. Rather, we are focusing on the effects of embedding in a strongly absorbing dispersive medium.

Past works have looked at the influence of geometry [36], background permittivity [37], and nanoparticle size [38] on the plasmon linewidth. We note that the past work on background permittivity suggested that for particles larger than $20 \mathrm{~nm}$, the linewidth increases when going to the infrared [37], which is the opposite of what we report. Compared to those works, we investigate the specific case of a surrounding lossy material where dispersion plays an important role in reducing the linewidth. We also present the first experimental findings showing the linewidth narrowing using such a strongly absorbing material as P3HT (poly(3-hexylthiophene)).

\section{EXPERIMENTAL METHODS}

Our experiments were performed by measuring the scattered power from illuminated samples on glass substrates. The samples consisted of gold nanoparticles encased in a thin layer of either P3HT or PMMA (poly(methyl methacrylate)). The nanoparticles used were $20 \mathrm{~nm}$ spherical gold nanoparticles $\left(1.1624 \times 10^{-12} \mathrm{mols} / \mathrm{mL}\right)$ purchased from BBI solutions. Drop coating these gold nanoparticles resulted in a $600 \mathrm{~nm}$ average inter-particle distance. The gold nanoparticles were randomly distributed after the drops evaporated. Clean glass 
(a)
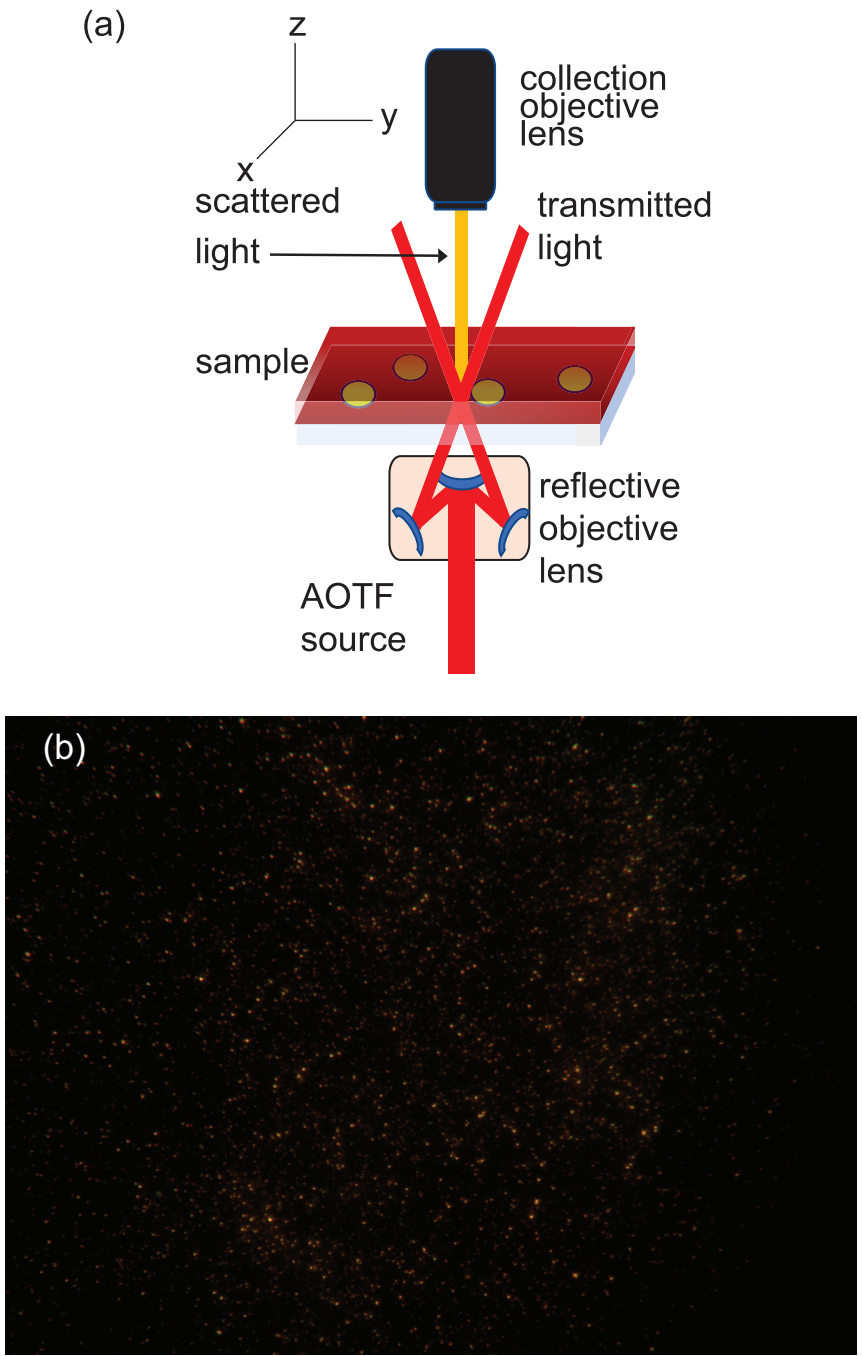

FIG. 1. (a) Schematic of the AOTF dark field microscope (b) $20 \mathrm{~nm}$ spherical gold nanoparticles drop coated on a glass slide and imaged with a CytoViva dark field microscope.

slides were prepared by sonication in an ethanol bath for 10 minutes, then sonication in an acetone bath for 10 minutes. Toluene was used to dissolve the solid P3HT. A $12 \mathrm{mg} / \mathrm{mL}$ P3HT:toluene solution mixed in a nitrogen atmosphere glovebox was spin-coated at $3500 \mathrm{RPM}$, with $10 \mathrm{~s}$ acceleration and deceleration time, and $20 \mathrm{~s}$ spin time to form a $100 \mathrm{~nm}$ thick layer [39]. To fully dissolve P3HT in toluene, solutions were placed on a hot plate for 30 minutes and stirred with a stir bar until they turned a bright orange color. The dissolved solutions were then filtered with a $0.2 \mu \mathrm{m}$ filter to remove any possible remaining P3HT clusters and other impurities. The P3HT solution was spin coated immediately upon removal from the heat source. For more information on the sample preparation, see Appendix A.

It has been shown that P3HT can form J-aggregate and $\mathrm{H}$-aggregate structures [40-42]; however, the spin coated layers of P3HT in the samples prepared were neither J- nor $\mathrm{H}$-aggregates as no characteristic resonance peak in the measured scattered power was observed in samples of P3HT alone; see Appendix F.
A dark field microscope was constructed to measure the light scattered by the samples. The schematic of the microscope is seen in Fig. 1(a). Figure 1(b) shows a dark field microscope image of $20 \mathrm{~nm}$ gold nanoparticles drop coated onto a clean glass slide. A supercontinuum laser (Fianium Supercontinuum SC450) provided incident power across the visible spectrum, while an acousto-optic tuneable filter (Fianium AOTF-DUAL) blocked most of the incident light, resulting in a $2-4 \mathrm{~nm}$ bandwidth at the desired wavelength. To obtain the scattering spectrum of the samples, a sweep of the incident wavelength from 500 to $700 \mathrm{~nm}$ was performed in $5 \mathrm{~nm}$ steps.

The AOTF dark field setup used a reflective objective lens (Thorlabs LMM-40x-P01, 40× magnification, 0.5 numerical aperture) which blocked the center of the incident light beam and excited at an angle of $30^{\circ}$. A collection objective lens ( $7 \times$ magnification and 0.2 numerical aperture) collected scattered light from $0^{\circ}$ to $12^{\circ}$. Detection was then performed with a Thorlabs Quantalux sCMOS (scientific complementary

(a)

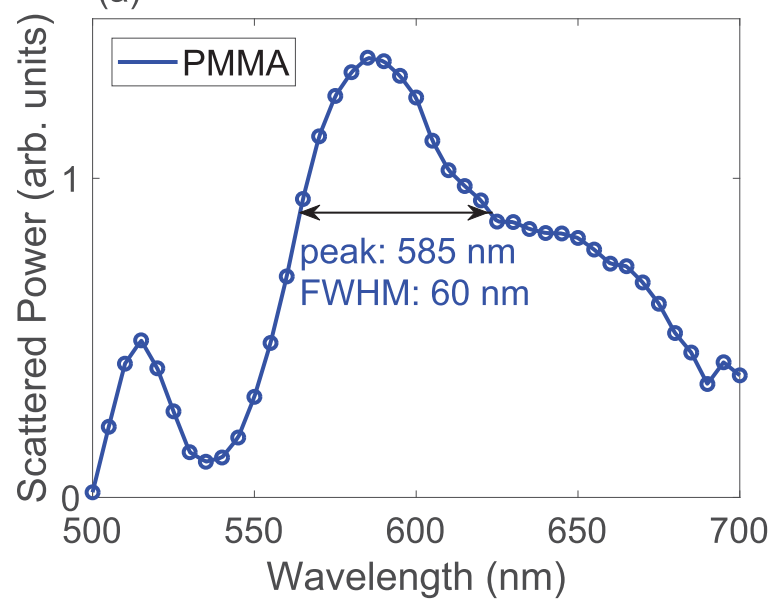

(b)

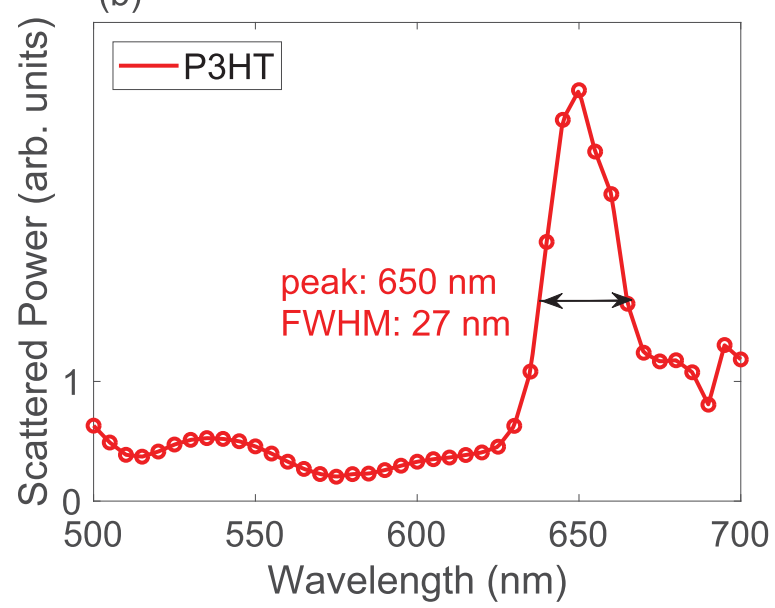

FIG. 2. Scattering data collected from the AOTF dark field microscope. The circles on the plots correspond to measured scattering data and occur in $5 \mathrm{~nm}$ steps. (a) Scattered power of $20 \mathrm{~nm}$ gold nanoparticles in PMMA. (b) Scattered power of $20 \mathrm{~nm}$ gold nanoparticles in P3HT. The position of the plasmon peak and the FWHM are displayed on each plot. 
metal-oxide-semiconductor) camera by integrating over the field of view; however, a simple detector could have been used as well.

Figure 2(a) shows the scattered power of $20 \mathrm{~nm}$ gold nanoparticles in PMMA and Fig. 2(b) shows the scattered power of $20 \mathrm{~nm}$ gold nanoparticles in P3HT obtained with the AOTF dark field microscope. The plasmon peak of gold nanoparticles in P3HT has less than half the linewidth of gold nanoparticles in PMMA.

\section{THEORY AND ANALYSIS}

Green function analysis (as well as finite-difference timedomain, FDTD, simulations shown in Appendix B) was performed in order to understand the observed linewidth narrowing for gold in P3HT. Figure 3(a) shows the Green function spectrum $S$, for a $20 \mathrm{~nm}$ gold nanoparticle in P3HT and PMMA for the case of an infinite and homogeneous surrounding medium. The scattering parameter $S$, shows stronger scattering for PMMA (prior to normalization, see Appendix H). The Green function spectrum for a single

(a)

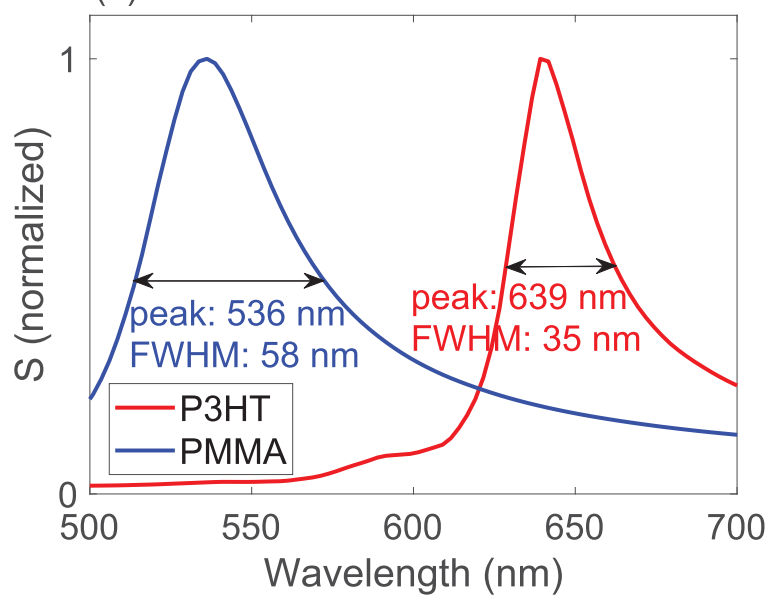

(b)

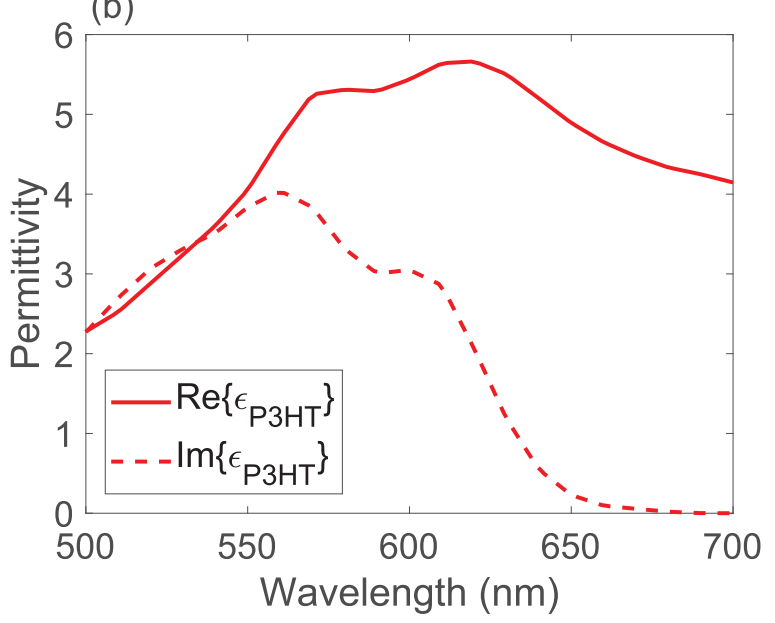

FIG. 3. (a) Green function spectrum $S$ for a single $20 \mathrm{~nm}$ gold nanoparticle in P3HT and PMMA. Surrounding media are assumed to be infinite and homogeneous. (b) Real and imaginary parts of the permittivity of P3HT and PMMA.

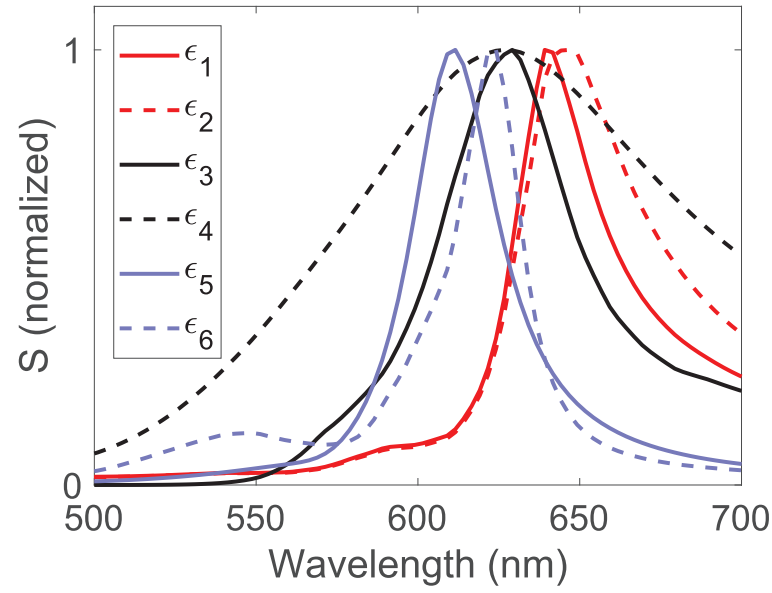

FIG. 4. Green function spectrum $S$ for a $20 \mathrm{~nm}$ gold nanoparticle in various infinite and homogeneous embedding media. The permittivities of the embedding media are $\epsilon_{1}=\epsilon_{\mathrm{P} 3 \mathrm{HT}}, \epsilon_{2}=5.2+$ $\operatorname{Im}\left\{\epsilon_{\mathrm{P} 3 \mathrm{HT}}\right\} i, \epsilon_{3}=\operatorname{Re}\left\{\epsilon_{\mathrm{P} 3 \mathrm{HT}}\right\}+1.5 i, \epsilon_{4}=5.2+1.5 i, \epsilon_{5}=5.2$ and $\epsilon_{6}=\operatorname{Re}\left\{\epsilon_{\mathrm{P} 3 \mathrm{HT}}\right\}$. The data are summarized in Table I.

spherical nanoparticle in a surrounding medium is given by

$$
S(\omega)=\left|\frac{1}{\epsilon_{0}} \mathbf{G}^{s}\left(\mathbf{r}, \mathbf{r}_{0}, \omega\right) \cdot \mathbf{d}\right|^{2}\left|\sqrt{\epsilon_{\text {med }}}\right|,
$$

where $\omega$ is the angular frequency of the source, $\epsilon_{0}$ is the permittivity of free space, $\epsilon_{\text {med }}$ is the relative permittivity of the surrounding medium, $\mathbf{G}^{s}$ is the scattered Green function, $\mathbf{r}$ is the location of the field point of interest, $\mathbf{r}_{0}$ is the location of the point dipole source, and $\mathbf{d}$ is the dipole moment, polarized along $z$. The Green function method goes beyond the common dipole approximation [43]; the resulting scattered field is exact within the context of Maxwell's equations. The derivation of the Green function spectrum in Eq. (1) is shown in Appendix G. The results for the linewidths for gold in PMMA and P3HT using the Green function spectra agree qualitatively with the AOTF dark field data from Figs. 2(a) and 2(b). The plasmon linewidth of gold in P3HT is half the linewidth of gold in PMMA.

Figure 3(b) shows the real and imaginary parts of the permittivity of P3HT and PMMA. The permittivity of PMMA is nearly constant from 500 to $700 \mathrm{~nm}$. The imaginary part of PMMA is almost zero over this wavelength range and so PMMA is considered lossless. Both the real and imaginary components of the permittivity of P3HT have large magnitudes and a large dispersion near the plasmon peak for gold in P3HT shown in Fig. 3(a).

To investigate how the embedding medium impacts the plasmon linewidth, calculations were performed for various background media. The effects of dispersion on the linewidth are not well documented in the literature. Most works assume there is no dispersion in the surrounding medium and this is usually a good approximation for commonly used embedding materials. We do not make this assumption in order to find the effects of the dispersion and loss on the plasmon peak. Figure 4 displays the Green function spectra for a single $20 \mathrm{~nm}$ gold nanoparticle in various homogeneous infinite embedding media. Table I shows the peak wavelengths and linewidths for 
TABLE I. Summary of plasmon peaks calculated from Green function spectra for $20 \mathrm{~nm}$ gold in various infinite and homogeneous embedding media. The scattering spectra for the permittivities listed in this table were calculated using Mie series expansions. When only five terms were used in the series expansions, the resulting linewidths were accurate to three decimal digits. The displayed linewidths were calculated using 23 terms and there is negligible numerical error in these values.

\begin{tabular}{lcrr}
\hline \hline Permittivity & Description & Peak & Linewidth \\
\hline$\epsilon_{1}=\epsilon_{\mathrm{P} 3 \mathrm{HT}}$ & actual material & $642 \mathrm{~nm}$ & 35 \\
$\epsilon_{2}=5.2+\operatorname{Im}\left\{\epsilon_{\mathrm{P} 3 \mathrm{HT}}\right\} i$ & dispersive imaginary component & $646 \mathrm{~nm}$ & 47 \\
$\epsilon_{3}=\operatorname{Re}\left\{\epsilon_{\mathrm{P} 3 \mathrm{HT}}+1.5 i\right.$ & dispersion, constant loss & $632 \mathrm{~nm}$ & 110 \\
$\epsilon_{4}=5.2+1.5 i$ & no dispersion, constant loss & $628 \mathrm{~nm}$ & 127 \\
$\epsilon_{5}=5.2$ & no dispersion, lossless & $613 \mathrm{~nm}$ & 33 \\
$\epsilon_{6}=\operatorname{Re}\left\{\epsilon_{\mathrm{P} 3 \mathrm{HT}}\right\}$ & dispersion, lossless & $624 \mathrm{~nm}$ & 22 \\
\hline \hline
\end{tabular}

each of the plasmon peaks in Fig. 4. We note that redshifting reduces the losses from interband transitions in gold [36] and the shift is sufficiently far to be slightly beyond the lossy peak of P3HT. Even so, in the following analysis, we break down the different contributions of the embedding medium permittivity to the linewidth, including dispersive effects in the real and imaginary parts of the medium permittivity.

Although the dielectric functions used are unphysical and do not obey the sum rules [44] and the Kramers-Kronig relations [1,2], we use them here simply to see how the magnitude of different parts of the permittivity impact the linewidth. The case of finite surrounding medium layers was analyzed with FDTD simulations and show there is only a small dependence of the plasmon peak on the layer thickness (for more details, see Appendix C). We also used a Green theory analysis to find how the size and permittivity of the metal nanoparticle affect the linewidth (Appendixes D and E). The Green theory analysis of the embedding medium permittivity indicated that four factors were important to the linewidths of the plasmons: the magnitude of both the real and imaginary parts of the permittivity, and the dispersion of the real and imaginary parts of the permittivity near the plasmon peak.

Dispersion in the imaginary part of the permittivity near the plasmon peak can result in linewidth narrowing. It can be seen from Fig. 4 that the left edges of the plasmon peaks for both $\epsilon_{1}=\epsilon_{\mathrm{P} 3 \mathrm{HT}}$ and $\epsilon_{2}=5.2+\operatorname{Im}\left(\epsilon_{\mathrm{P} 3 \mathrm{HT}}\right) i$ are the steepest. Near the plasmon peak, where the Fröhlich condition,

$$
\operatorname{Re}\left(\epsilon_{1}\right)=-2 \operatorname{Re}\left(\epsilon_{2}\right)
$$

for gold ( $\epsilon_{1}$ in the above formula) in P3HT ( $\epsilon_{2}$ in the above formula) is met $(611 \mathrm{~nm})$, the absorption of P3HT rapidly decreases which is reflected in the permittivity by a dispersive imaginary part. Table I shows that the lossless materials have narrower linewidths than the lossy materials. However, the presence of a dispersive loss component near the peak narrows the linewidth. Then an ideal medium for narrowing plasmon linewidths is one with a strongly dispersive imaginary component that rapidly goes to zero at the plasmon peak, not a lossless medium. This, however, is unphysical: changes in the real and imaginary parts of the permittivity are linked through the Kramers-Kronig relations [1,2]. P3HT still has some loss at the plasmon peak, and so it has a broader peak than the lossless materials. Although there is not very much absorption in $\mathrm{P} 3 \mathrm{HT}$ at the plasmon peak, there is still significant loss and dispersion near the peak. The presence of the large dispersion of the loss near the peak is a contributing factor to the narrow linewidth for gold in P3HT. The right edges of the plasmon peaks for $\epsilon_{1}=\epsilon_{\mathrm{P} 3 \mathrm{HT}}$ and $\epsilon_{2}=5.2+\operatorname{Im}\left(\epsilon_{\mathrm{P} 3 \mathrm{HT}}\right) i$ are not as steep due to the lack of a dispersive imaginary permittivity here. Therefore dispersion in the imaginary part of the permittivity of the surrounding medium can narrow linewidths.

Nondispersive loss leads to broader plasmon linewidths. To separate the effects of dispersion in the imaginary part of the permittivity from the magnitude of the imaginary part of the permittivity, the Green function spectra for background material permittivities with and without a nondispersive loss component were calculated. As seen in Fig. 4, the materials with constant loss $\left[\epsilon_{3}=\operatorname{Re}\left(\epsilon_{\mathrm{P} 3 \mathrm{HT}}\right)+1.5 i, \epsilon_{4}=\right.$ $5.2+1.5 i]$ have much broader linewidths than the materials with dispersive loss $\left[\epsilon_{1}=\epsilon_{\mathrm{P} 3 \mathrm{HT}}, \epsilon_{2}=5.2+\operatorname{Im}\left(\epsilon_{\mathrm{P} 3 \mathrm{HT}}\right) i\right]$ and with no loss. Therefore the dispersion of the loss narrows linewidths.

Dispersion in the real part of the medium permittivity, which arises near a lossy resonance, can also contribute to narrowing the linewidth. Comparing the plasmon peak for $\epsilon_{1}=\epsilon_{\mathrm{P} 3 \mathrm{HT}}$ and the peak for $\epsilon_{2}=5.2+\operatorname{Im}\left(\epsilon_{\mathrm{P} 3 \mathrm{HT}}\right) i$ in Fig. 4 , it is seen that the right edge of the plasmon peak for $\epsilon_{1}=$ $\epsilon_{\mathrm{P} 3 \mathrm{HT}}$ is steeper than the right edge of the peak for $\epsilon_{2}=$ $5.2+\operatorname{Im}\left(\epsilon_{\mathrm{P} 3 \mathrm{HT}}\right) i$. Figure $3(\mathrm{~b})$ shows that the real part of $\epsilon_{1}=\epsilon_{\mathrm{P} 3 \mathrm{HT}}$ decreases near the plasmon peak. The permittivity of gold becomes more negative for larger wavelengths (past $600 \mathrm{~nm}$ ). Then the Fröhlich condition [Eq. (2)] shows that at the resonance peak, decreasing $\epsilon_{1}$ and $\epsilon_{2}$ will result in a more rapid movement away from the resonance as the wavelength changes resulting in a narrower plasmon linewidth.

The magnitude of the real part of the permittivity can also narrow plasmon linewidths. In Fig. 4 , the $\epsilon_{5}=5.2$ peak yields a relatively narrow linewidth. The real permittivity of gold becomes more negative as well as more dispersive for larger wavelengths. Then for larger $\epsilon_{2}$ the Fröhlich condition [Eq. (2)] is satisfied at longer wavelengths. By redshifting the plasmon peak into the large dispersion regime of gold, the Fröhlich condition is swept through faster resulting in a narrower peak. Therefore a large real permittivity will narrow plasmon linewidths. The narrowest peak was obtained for $\epsilon_{6}=\operatorname{Re}\left(\epsilon_{\mathrm{P} 3 \mathrm{HT}}\right)$, which has both a large permittivity and large dispersion. The addition of the large dispersion to the permittivity causes a narrower peak than the $\epsilon_{5}=5.2$ peak. 


\section{CONCLUSION}

In conclusion, a narrow $27 \mathrm{~nm}(80 \mathrm{meV})$ plasmon linewidth has been observed in lossy P3HT with $20 \mathrm{~nm}$ gold nanoparticles using an experimental dark field setup. This linewidth was less than half the measured linewidth of $60 \mathrm{~nm}$ (220 meV) for the nonlossy PMMA with $20 \mathrm{~nm}$ gold nanoparticles. Using a Green function analysis of these systems, four factors were found to affect the plasmon linewidth: dispersion in the real permittivity, dispersion in the imaginary permittivity, magnitude of the real permittivity, and magnitude of the imaginary permittivity (loss). In contrast to past works $[5,35,45-48]$ which claim that losses must be reduced in plasmonic systems when narrow linewidths are desired, we have shown how including a lossy dispersive medium can serve to narrow plasmon linewidths. For metal nanoparticles with a decreasing permittivity (with respect to wavelength) near the plasmon peak, a decrease in the real and imaginary parts of the surrounding medium permittivity (normal dispersion) near the peak narrows the plasmon linewidth. A large magnitude of the real permittivity at the peak can narrow linewidths by redshifting the plasmon peak into a wavelength regime with larger dispersion. The addition of a nondispersive loss component broadens the plasmon peak. The large dispersion in both the real and imaginary parts of the permittivity for $\mathrm{P} 3 \mathrm{HT}$ as well as the large magnitude of the real part results in a narrow plasmon linewidth for gold nanoparticles in P3HT.

By using dispersive lossy materials, it may be possible to create enhanced plasmonic devices. Such a device may result from the combination of loss and gain media to make a nanolaser; dispersive lossy materials with embedded metal nanoparticles could be used to generate a narrow scattering response, while surrounding gain media could increase the power scattered at the peak wavelength. Dispersive lossy materials may also see applications in SPR sensing where a narrow linewidth is desirable, and allows for greater multiplexing within a given bandwidth [49-51].

\section{ACKNOWLEDGMENTS}

This work is supported by the Natural Sciences and Engineering Research Council of Canada Discovery Grants program and the Canadian Foundation for Innovation.

\section{APPENDIX A: SAMPLE PREPARATION}

Figures 5(a)-5(d) shows how the samples used in the AOTF dark field microscope were made. First a bottom layer of material (either P3HT or PMMA) was spin coated onto a clean glass slide, then $20 \mathrm{~nm}$ gold nanoparticles were drop coated with a micropipette on top of the bottom layer. The $50 \mu \mathrm{L}$ drops were heated at $90{ }^{\circ} \mathrm{C}$ for 10 minutes on a hot plate until the water in the drops evaporated, leaving gold nanoparticles on the surface of the sample. Finally, the top layer of material was spin coated onto the sample, encasing the gold nanoparticles. The PMMA solution used was $4 \%$ PMMA in anisole. P3HT was purchased from Rieke metals (4002-EE) and was $90 \%$ regioregular. (a)

(c)

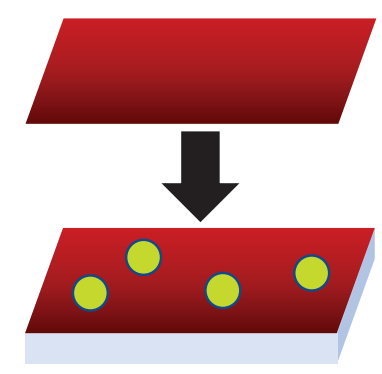

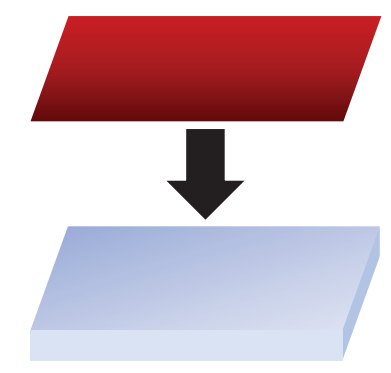

(b)

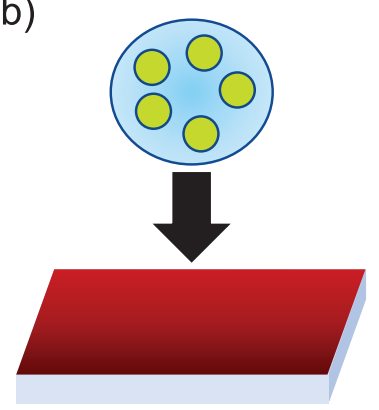

(d)

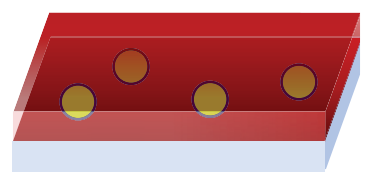

FIG. 5. [(a)-(d)] Steps followed to make samples. (a) Spin coat bottom layer. (b) Drop coat gold nanoparticles. (c) Spin coat top layer. (d) Encased gold nanoparticles.

\section{APPENDIX B: FDTD SIMULATION RESULTS}

Numerical calculations were performed using a commercial version (8.20.1731) of Lumerical FDTD (FiniteDifference Time-Domain). Figure 6(a) shows a schematic of the FDTD simulations. A total-field scattered-field (TFSF) source was used with a $50 \mathrm{~nm}$ side length and was incident on the sample at an angle of $30^{\circ}$, which is the incident angle used in the AOTF dark field setup. The wavelength of the source ranged from $400-800 \mathrm{~nm}$. The sample was modeled by a $120 \mathrm{~nm}$ thick rectangle of P3HT or PMMA, extending through the simulation boundaries with a single $20 \mathrm{~nm}$ gold nanoparticle at the center. The simulation region was a $1000 \mathrm{~nm}$ cube. PMLs (perfectly matched layers) were used at the boundaries and were 16-layer thick to more effectively absorb high angle scattered light. A single power monitor was placed outside the sample and the TFSF region to measure the scattered power. The gold permittivity used in the simulations was fitted to Johnson and Christy data [52], the P3HT permittivity was fitted to experimental data collected from the McGehee Stanford research group and is available online [53], and the PMMA permittivity was set to 2.25 . Convergence of all simulations was checked by gradually decreasing the mesh size down to a $1 \mathrm{~nm}$ mesh in the gold region and a $3 \mathrm{~nm}$ mesh in the surrounding medium.

Figure 6(b) shows the results of numerical simulations for the scattering cross section of $20 \mathrm{~nm}$ gold nanoparticles encased in $120 \mathrm{~nm}$ of PMMA and P3HT. A narrower linewidth is seen for the gold nanoparticles in the lossy medium. The calculated linewidths agree qualitatively with the scattering data from the AOTF dark field microscope shown in the main paper. 
(a)

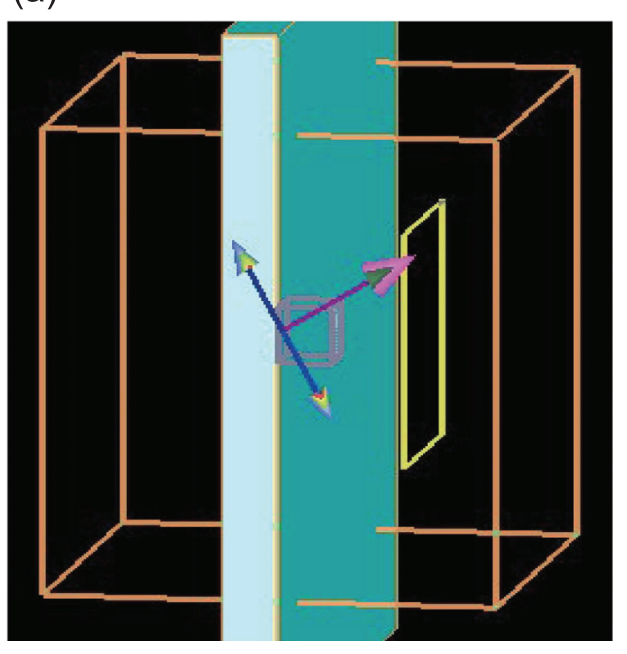

(b)

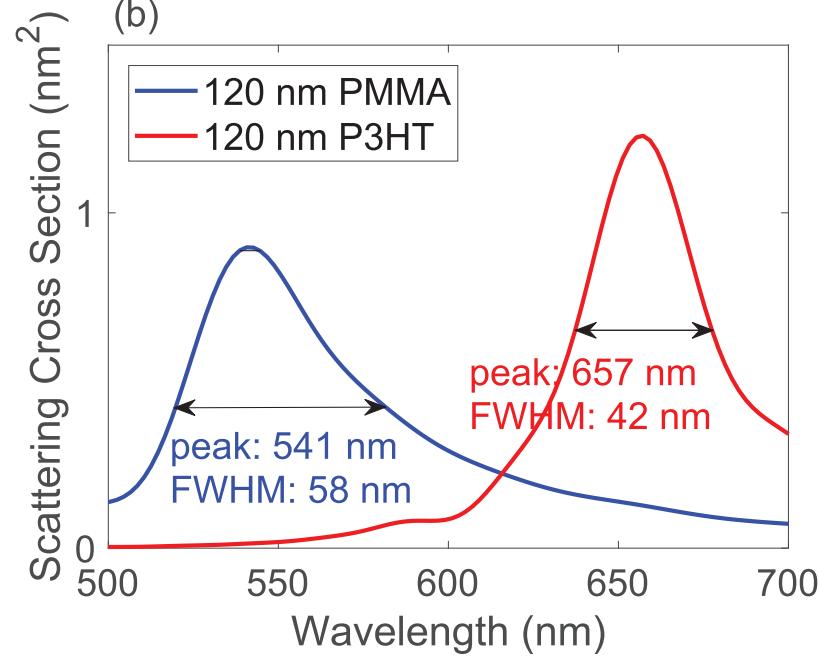

FIG. 6. Numerical simulation data. (a) A schematic of the Lumerical simulation. The blue rectangle is the $120 \mathrm{~nm}$ thick layer of PMMA, the large orange box is the simulation region, the small pink box is the TFSF source and the yellow square is the field monitor where the scattered field was measured. The $20 \mathrm{~nm}$ gold nanoparticle is in the center of the $120 \mathrm{~nm}$ film. (b) Scattering cross section calculated with FDTD from a $20 \mathrm{~nm}$ gold nanoparticle in $120 \mathrm{~nm}$ thick PMMA and from a $20 \mathrm{~nm}$ gold nanoparticle in $120 \mathrm{~nm}$ thick P3HT.

\section{APPENDIX C: FINITE THICKNESS EMBEDDING MEDIA}

Figures 7(a) and 7(b) show the results of FDTD simulations which found the scattering cross section of $20 \mathrm{~nm}$ gold nanoparticles embedded in media of varying thickness. These simulations were performed to find the dependence of the plasmon peak on the film thickness. Tables II and III display the peak locations and linewidths for each of these simulations. Table II shows that for PMMA, there is only a small change in the linewidth as the layer thickness is increased. Table III shows that the linewidth for P3HT remains the same over the range of thicknesses analyzed. For both PMMA and $\mathrm{P} 3 \mathrm{HT}$, the plasmon peak does not strongly depend on the thickness of the films used.

\section{APPENDIX D: NANOPARTICLE SIZE}

Figures 8(a) and 8(b) show the results of Green theory calculations for the normalized scattered power of a single gold nanoparticle in PMMA and in P3HT for different diameters

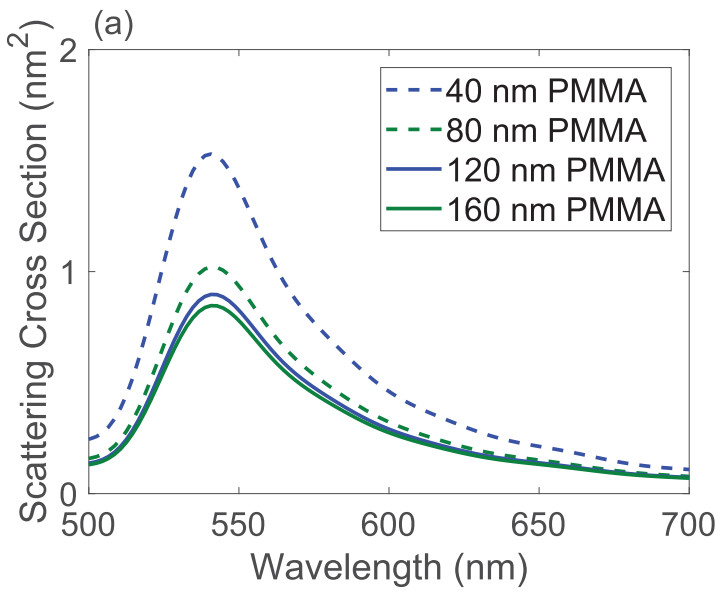

of the gold nanoparticle. Tables IV and V show the plasmon peak locations and linewidths. For both PMMA and P3HT, the plasmon peaks redshift and the linewidths broaden when the nanoparticle size is increased, so the narrowest linewidth occurs for the smallest nanoparticle. This linewidth broadening is caused by increased radiative damping (plasmon decay via coupling with the radiation field), as the particle size is increased [23,24]. For both PMMA and P3HT, the radiative damping becomes significant for a gold diameter of $30 \mathrm{~nm}$.

Surface scattering was not considered in this analysis. Surface scattering causes significant linewidth broadening for small nanoparticles $[27,28]$. If surface scattering was included, then the narrowest linewidth would occur for some intermediate particle size where the total damping was minimized.

\section{APPENDIX E: NANOPARTICLE PERMITTIVITY}

Figures 9(a) and 9(b) show the peaks from various goldlike metals embedded in infinite and homogeneous PMMA and

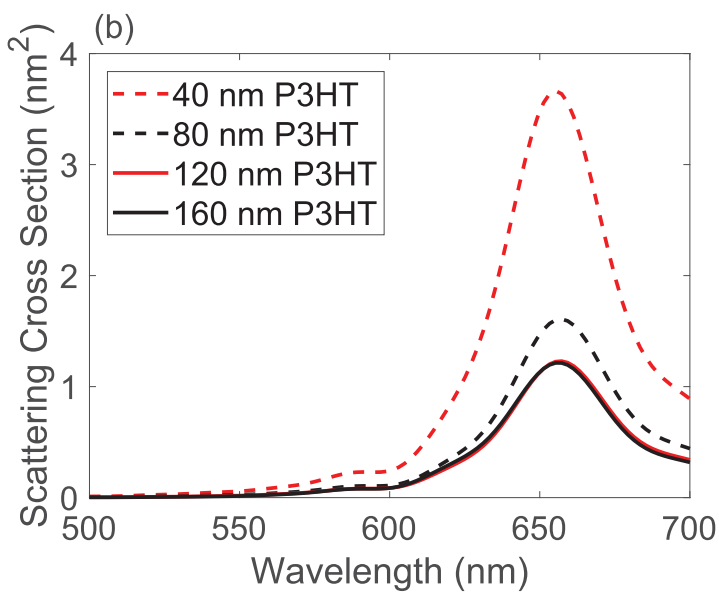

FIG. 7. (a) FDTD simulation results for the power scattered from a $20 \mathrm{~nm}$ gold nanoparticle embedded in PMMA films of varying thickness. (b) Similar FDTD simulation results for $20 \mathrm{~nm}$ gold in P3HT films. 
TABLE II. Summary of plasmon peak data from Fig. 7(a) for a $20 \mathrm{~nm}$ gold nanoparticle in layers of PMMA of various thickness.

\begin{tabular}{lcc}
\hline \hline Layer thickness & Peak & Linewidth \\
\hline $40 \mathrm{~nm}$ & $541 \mathrm{~nm}$ & $54 \mathrm{~nm}$ \\
$80 \mathrm{~nm}$ & $541 \mathrm{~nm}$ & $56 \mathrm{~nm}$ \\
$120 \mathrm{~nm}$ & $541 \mathrm{~nm}$ & $58 \mathrm{~nm}$ \\
$160 \mathrm{~nm}$ & $541 \mathrm{~nm}$ & $58 \mathrm{~nm}$ \\
\hline \hline
\end{tabular}

P3HT. Each calculation is done for a single $20 \mathrm{~nm}$ nanoparticle in either PMMA or P3HT. Tables VI and VII show the nanoparticle permittivities used, and the peak locations and linewidths for each calculation. Three factors significantly affected the linewidth.

Dispersion in the imaginary permittivity of the nanoparticle does not significantly narrow the peak. For plasmons excited with energies below the interband transition of gold $(2.4 \mathrm{eV}$ or $517 \mathrm{~nm})$ it is commonly assumed that the dispersion in the imaginary component is negligible compared to the dispersion in the real component, which we verify here $[54,55]$. Comparing $\epsilon_{1}=\epsilon_{\text {gold }}$ and $\epsilon_{5}=\operatorname{Re}\left\{\epsilon_{\text {gold }}\right\}+1.02 i$ in Fig. 9(a) which have equal linewidths, and also $\epsilon_{1}=\epsilon_{\mathrm{P} 3 \mathrm{HT}}$ and $\epsilon_{5}=$ $\operatorname{Re}\left\{\epsilon_{\text {gold }}\right\}+1.02 i$ in Fig. 9(b) which have equal linewidths, we see the inclusion of the dispersive imaginary part to the nanoparticle permittivity does not change the plasmon peak significantly. Unlike for the surrounding medium analysis in the main paper, there is no steep edge in the plasmon peak due to the dispersive imaginary permittivity of gold. The real permittivity of gold changes much more rapidly than the imaginary permittivity at the plasmon peaks for PMMA and P3HT. Although the dispersion in the imaginary permittivity can alter the plasmon peak very slightly and may cause some degree of linewidth narrowing, it is insignificant compared to the narrowing due to the large real permittivity dispersion. To isolate the effects of dispersion from only the imaginary part of the permittivity, we can compare the results for $\epsilon_{4}=$ $-13.3+\operatorname{Im}\left\{\epsilon_{\text {gold }}\right\} i$ and $\epsilon_{6}=-13.3+1.02 i$ from Fig. 9(b). The inclusion of the dispersive imaginary part of gold only
TABLE III. Summary of plasmon peak data from Fig. 7(b) for a $20 \mathrm{~nm}$ gold nanoparticle in layers of P3HT of various thickness.

\begin{tabular}{lcc}
\hline \hline Layer thickness & Peak & Linewidth \\
\hline $40 \mathrm{~nm}$ & $655 \mathrm{~nm}$ & $42 \mathrm{~nm}$ \\
$80 \mathrm{~nm}$ & $657 \mathrm{~nm}$ & $42 \mathrm{~nm}$ \\
$120 \mathrm{~nm}$ & $657 \mathrm{~nm}$ & $42 \mathrm{~nm}$ \\
$160 \mathrm{~nm}$ & $655 \mathrm{~nm}$ & $42 \mathrm{~nm}$ \\
\hline \hline
\end{tabular}

slightly reduces the linewidth by $1 \mathrm{~nm}$ from 68 to $67 \mathrm{~nm}$. Also, both $\epsilon_{6}=-5.23+1.97 i$ and $\epsilon_{4}=-5.23+\operatorname{Im}\left\{\epsilon_{\text {gold }}\right\} i$ shown in Fig. 9(a) do not have plasmon peaks. If the effects of the dispersive imaginary permittivity were significant, there would be a plasmon peak for $\epsilon_{4}=-5.23+\operatorname{Im}\left\{\epsilon_{\text {gold }}\right\} i$. Therefore, for a gold nanoparticle, the dispersion in the imaginary permittivity does not contribute significantly to narrowing plasmon linewidths for plasmons below the interband transitions.

Nondispersive loss in the nanoparticle broadens the linewidth. In Fig. $9(a), \epsilon_{2}=\operatorname{Re}\left\{\epsilon_{\text {gold }}\right\}$ has an extremely narrow $2 \mathrm{~nm}$ peak while the same permittivity with loss included, $\epsilon_{5}=\operatorname{Re}\left\{\epsilon_{\text {gold }}\right\}+1.97 i$, has a much broader linewidth of $58 \mathrm{~nm}$. In Fig. 9(b), $\epsilon_{2}=\operatorname{Re}\left\{\epsilon_{\text {gold }}\right\}$ has a $27 \mathrm{~nm}$ linewidth and the permittivity $\epsilon_{5}=\operatorname{Re}\left\{\epsilon_{\text {gold }}\right\}+1.02 i$ has a broader $35 \mathrm{~nm}$ linewidth. The linewidth is broadened by adding a nondispersive loss component to the nanoparticle permittivity. The permittivity $\epsilon_{2}=\operatorname{Re}\left\{\epsilon_{\text {gold }}\right\}$ in PMMA has such a narrow peak because PMMA is also assumed to have no loss, so when the Fröhlich condition is met the scattered power becomes infinite in the quasistatic regime. The reason the peak is not infinite here is because the Green theory does not use the quasistatic approximation, so retardation of the field in the nanoparticle leads to radiation damping which will broaden the peak and reduce the scattered power to a finite number. Although having no loss is unphysical, this investigation shows the effectiveness of reducing loss in the nanoparticle for narrowing linewidths. (a)

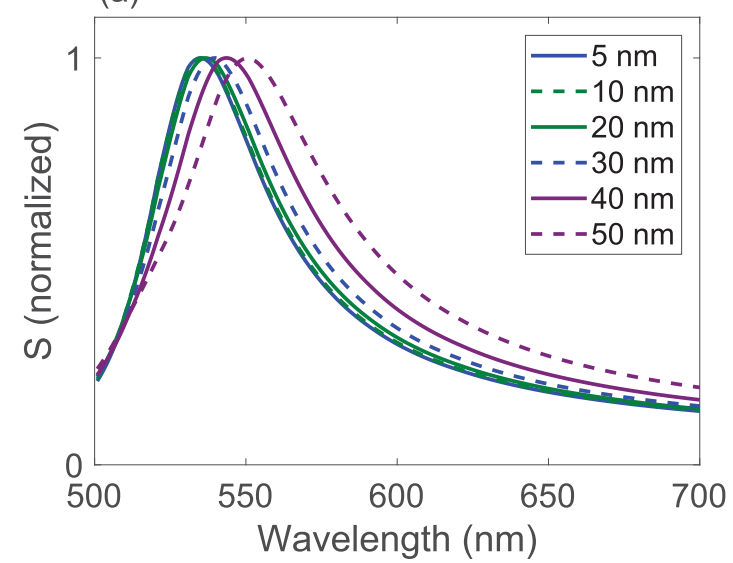

(b)

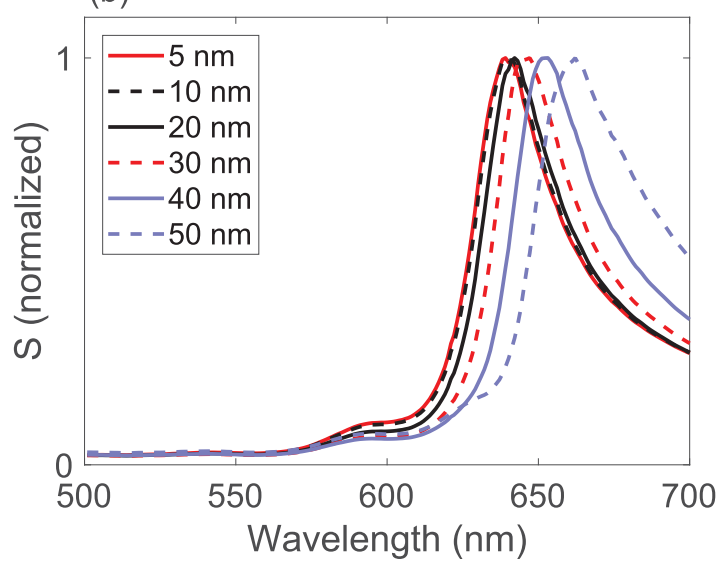

FIG. 8. (a) Green function calculations for the power scattered (spectrum) from gold nanoparticles of varying diameter embedded in an infinite and homogeneous PMMA medium. (b) Similar calculations for gold in P3HT. In each plot, the numbers in the legend display the gold nanoparticle diameters. 
TABLE IV. Summary of plasmon peak data from Fig. 8(a) for different size gold nanoparticles in PMMA.

\begin{tabular}{lcc}
\hline \hline Nanoparticle size & Peak & Linewidth \\
\hline $5 \mathrm{~nm}$ & $534 \mathrm{~nm}$ & $56 \mathrm{~nm}$ \\
$10 \mathrm{~nm}$ & $534 \mathrm{~nm}$ & $57 \mathrm{~nm}$ \\
$20 \mathrm{~nm}$ & $536 \mathrm{~nm}$ & $58 \mathrm{~nm}$ \\
$30 \mathrm{~nm}$ & $539 \mathrm{~nm}$ & $60 \mathrm{~nm}$ \\
$40 \mathrm{~nm}$ & $542 \mathrm{~nm}$ & $67 \mathrm{~nm}$ \\
$50 \mathrm{~nm}$ & $550 \mathrm{~nm}$ & $76 \mathrm{~nm}$ \\
\hline
\end{tabular}

Dispersion in the real part of the nanoparticle permittivity can narrow linewidths. In Fig. 9(a), $\epsilon_{1}=\epsilon_{\text {gold }}$ has a peak of $58 \mathrm{~nm}$ while there is no plasmon peak for $\epsilon_{4}=-5.23+$ $\operatorname{Im}\left\{\epsilon_{\text {gold }}\right\} i$. In Fig. 9(b), $\epsilon_{1}=\epsilon_{\text {gold }}$ has a linewidth of $35 \mathrm{~nm}$ and $\epsilon_{4}=-13.3+\operatorname{Im}\left\{\epsilon_{\text {gold }}\right\} i$ has a much broader linewidth of $67 \mathrm{~nm}$. The constant values -5.23 and -13.3 for $\epsilon_{4}$ in each medium were chosen so that $\epsilon_{4}=\epsilon_{1}$ at the plasmon peak. Thus, the drastic changes in linewidth are due to the large dispersion in the real permittivity of gold, so dispersion in the real permittivity can narrow plasmon linewidths.

A large magnitude of the real permittivity of the nanoparticle can narrow linewidths. In Figs. 9(a) and 9(b), $\epsilon_{3}=$ $\operatorname{Im}\left\{\epsilon_{\text {gold }}\right\} i$ do not have plasmon peaks because there is no negative real permittivity (the real component of the permittivity is set to 0). As the magnitude of the permittivity increases to the value at the plasmon peak, the peak gradually narrows. The magnitude of the real permittivity of the metal nanoparticle determines where the plasmon peak is located in the electromagnetic spectrum. Metals commonly used in nanoplasmonics, such as gold and silver, have a large dispersive real permittivity below the plasma frequency. Therefore a large magnitude in the nanoparticle permittivity can narrow linewidths by shifting the plasmon peak into a regime where there is a large dispersion in the real permittivity of the metal.

\section{APPENDIX F: P3HT SCATTERING DATA}

Figure 10 shows the scattering data from a $120 \mathrm{~nm}$ layer of P3HT without any gold nanoparticles. The measurements were taken using the same AOTF dark field microscope described in the main paper. There was no narrow peak at $650 \mathrm{~nm}$ as there was for $120 \mathrm{~nm}$ P3HT with embedded $20 \mathrm{~nm}$ gold nanoparticles. These data show that the $650 \mathrm{~nm}$ scattering peak present for gold in P3HT is not due to fluorescence or scattering from the P3HT layer.

TABLE V. Summary of plasmon peak data from Fig. 8(b) for a different size gold nanoparticles in P3HT.

\begin{tabular}{lcc}
\hline \hline Nanoparticle size & Peak & Linewidth \\
\hline $5 \mathrm{~nm}$ & $636 \mathrm{~nm}$ & $35 \mathrm{~nm}$ \\
$10 \mathrm{~nm}$ & $636 \mathrm{~nm}$ & $35 \mathrm{~nm}$ \\
$20 \mathrm{~nm}$ & $639 \mathrm{~nm}$ & $35 \mathrm{~nm}$ \\
$30 \mathrm{~nm}$ & $644 \mathrm{~nm}$ & $37 \mathrm{~nm}$ \\
$40 \mathrm{~nm}$ & $650 \mathrm{~nm}$ & $41 \mathrm{~nm}$ \\
$50 \mathrm{~nm}$ & $659 \mathrm{~nm}$ & $52 \mathrm{~nm}$ \\
\hline \hline
\end{tabular}

(a)

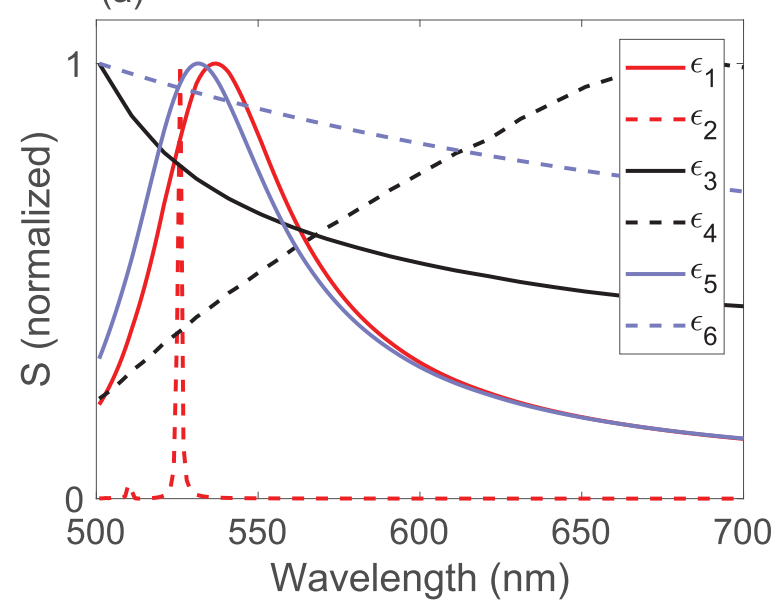

(b)

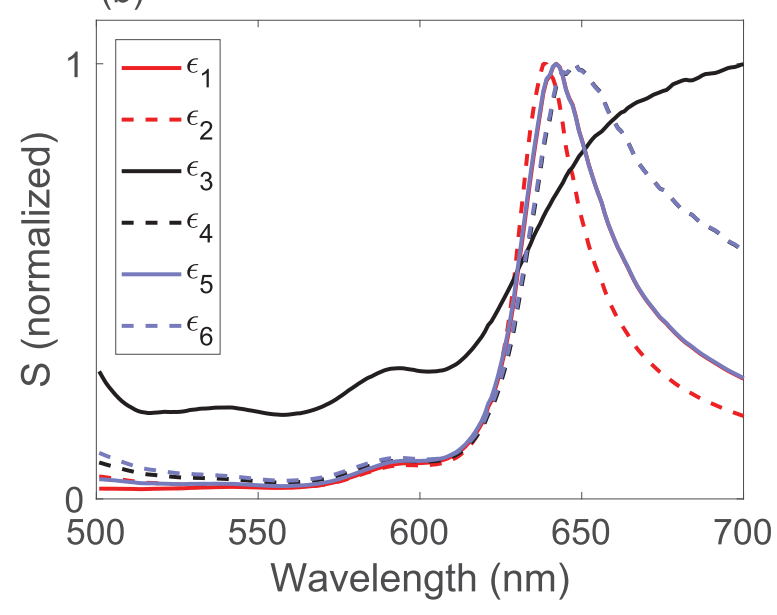

FIG. 9. (a) Green function spectrum $S$ for different $20 \mathrm{~nm}$ metal nanoparticles embedded in infinite and homogeneous PMMA. (b) Green function spectrum $S$ for different $20 \mathrm{~nm}$ metal nanoparticles embedded in infinite and homogeneous P3HT. Tables VI and VII show the metal nanoparticle permittivities used and summarize the results shown in these figures.

\section{APPENDIX G: GREEN FUNCTION ANALYSIS}

The Green function for Maxwell's equations satisfies

$$
\begin{aligned}
\nabla & \times \nabla \times \mathbf{G}\left(\mathbf{r}, \mathbf{r}_{0}, \omega\right)-\frac{\omega^{2}}{c^{2}} \epsilon(\mathbf{r}, \omega) \mathbf{G}\left(\mathbf{r}, \mathbf{r}_{0}, \omega\right) \\
& =\frac{\omega^{2}}{c^{2}} \mathbf{1} \delta\left(\mathbf{r}-\mathbf{r}_{0}\right),
\end{aligned}
$$

where $\mathbf{G}$ is the dyadic Green function, $\omega$ is the angular frequency, $\mathbf{r}$ is the location of the field point of interest, $\mathbf{r}_{0}$ is the location of the source, $c$ is the speed of light, $\epsilon(\mathbf{r}, \omega)$ is the permittivity, $\mathbf{1}$ is the unit tensor, and $\delta$ is the Dirac delta function. The full Green function is split into scattering and homogeneous medium contributions

$$
\mathbf{G}=\mathbf{G}^{s}+\mathbf{G}^{\text {hom }} .
$$

The formula for the scattering contribution of the Green function, $\mathbf{G}^{s}$, can be found in a past work [43]. The Green 
TABLE VI. Summary of plasmon peaks calculated from Green function spectra for various gold-like metals in infinite and homogeneous PMMA. A row marked with " $X$ " means there was no plasmon peak for this calculation. The constant values of -5.23 and $1.97 \mathrm{i}$ were used because at the plasmon peak for gold and PMMA the permittivity of gold is $\epsilon_{\text {gold }}=-5.23+1.97 i$.

\begin{tabular}{lcc}
\hline \hline Permittivity & Peak & Linewidth \\
\hline$\epsilon_{1}=\epsilon_{\text {gold }}$ & $536 \mathrm{~nm}$ & $58 \mathrm{~nm}$ \\
$\epsilon_{2}=\operatorname{Re}\left\{\epsilon_{\text {gold }}\right\}$ & $525 \mathrm{~nm}$ & $2 \mathrm{~nm}$ \\
$\epsilon_{3}=\operatorname{Im}\left\{\epsilon_{\text {gold }}\right\} i$ & $\mathrm{X}$ & $\mathrm{X}$ \\
$\epsilon_{4}=-5.23+\operatorname{Im}\left\{\epsilon_{\text {gold }}\right\} i$ & $\mathrm{X}$ & $\mathrm{X}$ \\
$\epsilon_{5}=\operatorname{Re}\left\{\epsilon_{\text {gold }}\right\}+1.97 i$ & $531 \mathrm{~nm}$ & $58 \mathrm{~nm}$ \\
$\epsilon_{6}=-5.23+1.97 i$ & $\mathrm{X}$ & $\mathrm{X}$ \\
\hline \hline
\end{tabular}

function spectrum is defined as

$$
S_{0}(\omega)=\left|\frac{1}{\epsilon_{0}} \mathbf{G}^{s}\left(\mathbf{r}, \mathbf{r}_{0}, \omega\right) \cdot \mathbf{d}\right|^{2},
$$

where $\mathbf{G}^{s}$ is the scattering part of the Green function at position $\mathbf{r}$ from the center of the nanoparticle, and the source is a dipole polarized in the $z$ direction located at $\mathbf{r}_{0}$ with dipole moment $\mathbf{d}$ (the $z$ axis is defined to be the direction from the center of the nanoparticle to the dipole point $\mathbf{r}_{0}$, and the point $\mathbf{r}$ is further up the same axis). The Green function spectrum defined here quantifies the power scattered by the nanoparticle.

The calculations were performed with the distance between the field point and the dipole $\left|\mathbf{r}-\mathbf{r}_{0}\right|$ set to $50 \mathrm{~nm}$. This gives similar loss from the medium as in the thin film calculations and experiments. The far field scattered power in an infinite homogeneous lossy medium is not a well-defined property as the spectral shape is continuously changing as the distance the scattered light travels through the lossy medium is increased, and so the far field scattered power was not calculated.

In experiments, the incident light crossed from air to the surrounding medium, while in calculations the light from the source was generated inside the surrounding medium. To account for this difference the scattered power was

TABLE VII. Summary of plasmon peaks calculated from Green function spectra for various gold-like metals in infinite and homogeneous P3HT. A row marked with " $\mathrm{X}$ " means there was no plasmon peak for this calculation. The constant values of -13.3 and $1.02 \mathrm{i}$ were used because at the plasmon peak for gold and P3HT the permittivity of gold is $\epsilon_{\text {gold }}=-13.3+1.02 i$.

\begin{tabular}{lcc}
\hline \hline Permittivity & Peak & Linewidth \\
\hline$\epsilon_{1}=\epsilon_{\text {gold }}$ & $639 \mathrm{~nm}$ & $35 \mathrm{~nm}$ \\
$\epsilon_{2}=\operatorname{Re}\left\{\epsilon_{\text {gold }}\right\}$ & $635 \mathrm{~nm}$ & $27 \mathrm{~nm}$ \\
$\epsilon_{3}=\operatorname{Im}\left\{\epsilon_{\text {gold }}\right\} i$ & $\mathrm{X}$ & $\mathrm{X}$ \\
$\epsilon_{4}=-13.3+\operatorname{Im}\left\{\epsilon_{\text {gold }}\right\} i$ & 647 & 67 \\
$\epsilon_{5}=\operatorname{Re}\left\{\epsilon_{\text {gold }}\right\}+1.02 i$ & $639 \mathrm{~nm}$ & $35 \mathrm{~nm}$ \\
$\epsilon_{6}=-13.3+1.02 i$ & 647 & 68 \\
\hline \hline
\end{tabular}

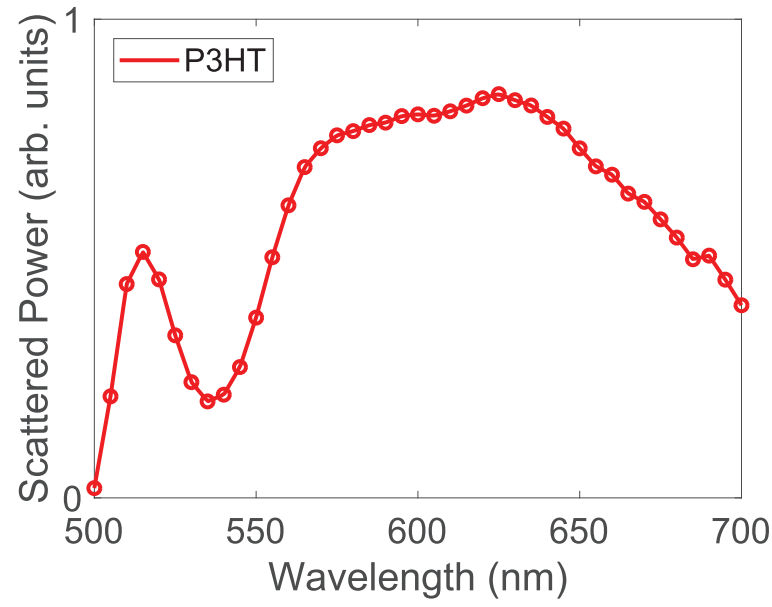

FIG. 10. Scattered power spectrum from a $120 \mathrm{~nm}$ layer of P3HT. The red circles are experimental measurements taken in $5 \mathrm{~nm}$ increments.

renormalized:

$$
S=S_{0}\left|\sqrt{\epsilon_{\text {med }}}\right|
$$

where $\epsilon_{\text {med }}$ was the medium permittivity.

\section{APPENDIX H: UNNORMALIZED SCATTERING SPECTRA}

Figure 11 shows the scattering without normalization. Similar results were obtained using numerical simulations with COMSOL, however accounting for the propagation through the absorbing medium complicates the analysis.

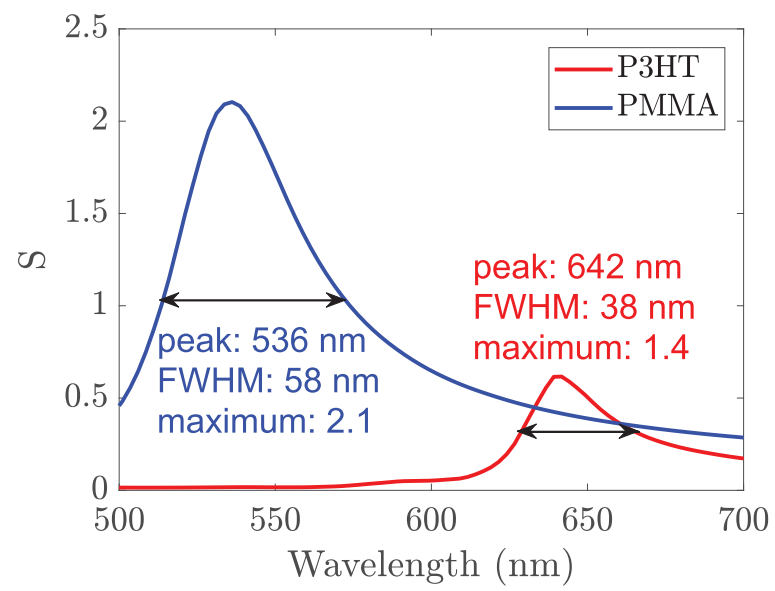

FIG. 11. Green theory scattered power for PMMA and P3HT without normalization. 
[1] R. d. L. Kronig, On the theory of dispersion of x-rays, J. Opt. Soc. Am. A 12, 547 (1926).

[2] H. A. Kramers, La diffusion de la lumiere par les atomes, in Atti Cong. Intern. Fisica (Transactions of Volta Centenary Congress) Como (1927), Vol. 2, pp. 545-557.

[3] R. P. Feynman, R. B. Leighton, and M. Sands, The Feynman Lectures on Physics, Vol. I: The New Millennium Edition: Mainly Mechanics, Radiation, and Heat (Basic books, 2011), Vol. 1.

[4] N. Engheta, A. Salandrino, and A. Alu, Circuit Elements at Optical Frequencies: Nanoinductors, Nanocapacitors, and Nanoresistors, Phys. Rev. Lett. 95, 095504 (2005).

[5] S. A. Maier, Plasmonics: Fundamentals and Applications (Springer Science \& Business Media, 2007).

[6] C. F. Bohren and D. P. Gilra, Extinction by a spherical particle in an absorbing medium, J. Colloid Interface Sci. 72, 215 (1979).

[7] G. Videen and W. Sun, Yet another look at light scattering from particles in absorbing media, Appl. Opt. 42, 6724 (2003).

[8] M. I. Mishchenko, Electromagnetic scattering by a fixed finite object embedded in an absorbing medium, Opt. Express 15, 13188 (2007).

[9] J. C. Ndukaife, V. M. Shalaev, and A. Boltasseva, Plasmonicsturning loss into gain, Science 351, 334 (2016).

[10] I. De Leon and P. Berini, Spontaneous emission in longrange surface plasmon-polariton amplifiers, Phys. Rev. B 83, 081414(R) (2011).

[11] C. Billaudeau, S. Collin, F. Pardo, N. Bardou, and J.-L. Pelouard, Tailoring radiative and non-radiative losses of thin nanostructured plasmonic waveguides, Opt. Express 17, 3490 (2009).

[12] A. Marini, D. V. Skryabin, and B. Malomed, Stable spatial plasmon solitons in a dielectric-metal-dielectric geometry with gain and loss, Opt. Express 19, 6616 (2011).

[13] A. D. Boardman, V. V. Grimalsky, Y. S. Kivshar, S. V. Koshevaya, M. Lapine, N. M. Litchinitser, V. N. Malnev, M. Noginov, Y. G. Rapoport, and V. M. Shalaev, Active and tunable metamaterials, Laser Photonics Rev. 5, 287 (2011).

[14] O. Hess, J. B. Pendry, S. A. Maier, R. F. Oulton, J. M. Hamm, and K. L. Tsakmakidis, Active nanoplasmonic metamaterials, Nat. Mater. 11, 573 (2012).

[15] F. D. Nunes, T. C. Vasconcelos, M. Bezerra, and J. Weiner, Electromagnetic energy density in dispersive and dissipative media, J. Opt. Soc. Am. B 28, 1544 (2011).

[16] A. Reza, M. Dignam, and S. Hughes, Can light be stopped in realistic metamaterials? Nature 455, E10 (2008).

[17] T. K. Gaylord, E. N. Glytsis, and M. Moharam, Zero-reflectivity homogeneous layers and high spatial-frequency surface-relief gratings on lossy materials, Appl. Opt. 26, 3123 (1987).

[18] M. S. Tame, K. McEnery, Ş. Özdemir, J. Lee, S. A. Maier, and M. Kim, Quantum plasmonics, Nat. Phys. 9, 329 (2013).

[19] A. F. Koenderink, Single-photon nanoantennas, ACS photonics 4, 710 (2017).

[20] S. Hughes, S. Franke, C. Gustin, M. Kamandar Dezfouli, A. Knorr, and M. Richter, Theory and limits of on-demand single-photon sources using plasmonic resonators: A quantized quasinormal mode approach, ACS Photonics 6, 2168 (2019).

[21] A. I. Fernández-Domínguez, S. I. Bozhevolnyi, and N. A. Mortensen, Plasmon-enhanced generation of nonclassical light, Acs Photonics 5, 3447 (2018).
[22] S. Franke, M. Richter, J. Ren, A. Knorr, and S. Hughes, Quantized quasinormal mode description of non-linear cavity QED effects from coupled resonators with a Fano-like resonance, Phys. Rev. Research 2, 033456 (2020).

[23] A. Wokaun, J. P. Gordon, and P. F. Liao, Radiation Damping in Surface-Enhanced Raman Scattering, Phys. Rev. Lett. 48, 957 (1982).

[24] T. Kokkinakis and K. Alexopoulos, Observation of Radiative Decay of Surface Plasmons in Small Silver Particles, Phys. Rev. Lett. 28, 1632 (1972).

[25] A.-L. Baudrion, F. de Leon-Perez, O. Mahboub, A. Hohenau, H. Ditlbacher, F. J. Garcia-Vidal, J. Dintinger, T. W. Ebbesen, L. Martin-Moreno, and J. R. Krenn, Coupling efficiency of light to surface plasmon polariton for single subwavelength holes in a gold film, Opt. Express 16, 3420 (2008).

[26] X. Guo, Y. Ma, Y. Wang, and L. Tong, Nanowire plasmonic waveguides, circuits and devices, Laser Photonics Rev. 7, 855 (2013).

[27] L. Genzel, T. Martin, and U. Kreibig, Dielectric function and plasma resonances of small metal particles, Z. Phys. B 21, 339 (1975).

[28] U. Kreibig and L. Genzel, Optical absorption of small metallic particles, Surf. Sci. 156, 678 (1985).

[29] H. Hövel, S. Fritz, A. Hilger, U. Kreibig, and M. Vollmer, Width of cluster plasmon resonances: Bulk dielectric functions and chemical interface damping, Phys. Rev. B 48, 18178 (1993).

[30] B. Foerster, A. Joplin, K. Kaefer, S. Celiksoy, S. Link, and C. Sönnichsen, Chemical interface damping depends on electrons reaching the surface, ACS nano 11, 2886 (2017).

[31] T. V. Shahbazyan, Landau damping of surface plasmons in metal nanostructures, Phys. Rev. B 94, 235431 (2016).

[32] J. Khurgin, W.-Y. Tsai, D. P. Tsai, and G. Sun, Landau damping and limit to field confinement and enhancement in plasmonic dimers, ACS Photonics 4, 2871 (2017).

[33] J. P. Marton and B. D. Jordan, Optical properties of aggregated metal systems: Interband transitions, Phys. Rev. B 15, 1719 (1977).

[34] S. Link and M. A. El-Sayed, Spectral properties and relaxation dynamics of surface plasmon electronic oscillations in gold and silver nanodots and nanorods, J. Phys. Chem. B 103, 8410 (1999).

[35] T. Klar, M. Perner, S. Grosse, G. Von Plessen, W. Spirkl, and J. Feldmann, Surface-Plasmon Resonances in Single Metallic Nanoparticles, Phys. Rev. Lett. 80, 4249 (1998).

[36] C. Novo, D. Gomez, J. Perez-Juste, Z. Zhang, H. Petrova, M. Reismann, P. Mulvaney, and G. V. Hartland, Contributions from radiation damping and surface scattering to the linewidth of the longitudinal plasmon band of gold nanorods: A single particle study, Phys. Chem. Chem. Phys. 8, 3540 (2006).

[37] M. Hu, C. Novo, A. Funston, H. Wang, H. Staleva, S. Zou, P. Mulvaney, Y. Xia, and G. V. Hartland, Dark-field microscopy studies of single metal nanoparticles: Understanding the factors that influence the linewidth of the localized surface plasmon resonance, J. Mater. Chem. 18, 1949 (2008).

[38] M. M. Alvarez, J. T. Khoury, T. G. Schaaff, M. N. Shafigullin, I. Vezmar, and R. L. Whetten, Optical absorption spectra of nanocrystal gold molecules, J. Phys. Chem. B 101, 3706 (1997).

[39] L. Janasz, D. Chlebosz, M. Gradzka, W. Zajaczkowski, T. Marszalek, K. Müllen, J. Ulanski, A. Kiersnowski, and W. Pisula, Improved charge carrier transport in ultrathin poly 
(3-hexylthiophene) films via solution aggregation, J. Mater. Chem. C 4, 11488 (2016).

[40] F. C. Spano and C. Silva, H-and J-aggregate behavior in polymeric semiconductors, Annu. Rev. Phys. Chem. 65, 477 (2014).

[41] M. Baghgar, J. A. Labastide, F. Bokel, R. C. Hayward, and M. D. Barnes, Effect of polymer chain folding on the transition from h-to j-aggregate behavior in p3ht nanofibers, J. Phys. Chem. C 118, 2229 (2014).

[42] E. T. Niles, J. D. Roehling, H. Yamagata, A. J. Wise, F. C. Spano, A. J. Moulé, and J. K. Grey, J-aggregate behavior in poly-3-hexylthiophene nanofibers, J. Phys. Chem. Lett. 3, 259 (2012).

[43] C. Van Vlack, P. T. Kristensen, and S. Hughes, Spontaneous emission spectra and quantum light-matter interactions from a strongly coupled quantum dot metal-nanoparticle system, Phys. Rev. B 85, 075303 (2012).

[44] M. Dapor, Appendix G: The Kramers-Kronig relations and the sum rules, in Transport of Energetic Electrons in Solids (Springer, 2017), pp. 175-178.

[45] C. Sönnichsen, T. Franzl, T. Wilk, G. Von Plessen, and J. Feldmann, Plasmon resonances in large noble-metal clusters, New J. Phys. 4, 93 (2002).

[46] P. R. West, S. Ishii, G. V. Naik, N. K. Emani, V. M. Shalaev, and A. Boltasseva, Searching for better plasmonic materials, Laser Photonics Rev. 4, 795 (2010).
[47] J. B. Khurgin, How to deal with the loss in plasmonics and metamaterials, Nat. Nanotechnol. 10, 2 (2015).

[48] M. I. Mishchenko and J. M. Dlugach, Plasmon resonances of metal nanoparticles in an absorbing medium, OSA Continuum 2, 3415 (2019).

[49] C. Yu and J. Irudayaraj, Multiplex biosensor using gold nanorods, Anal. Chem. (Washington, DC, US) 79, 572 (2007).

[50] J. Dostálek, H. Vaisocherová, and J. Homola, Multichannel surface plasmon resonance biosensor with wavelength division multiplexing, Sens. Actuators, B 108, 758 (2005).

[51] R. Sobral-Filho, A. Brito-Silva, M. Isabelle, A. Jirasek, J. Lum, and A. Brolo, Plasmonic labeling of subcellular compartments in cancer cells: Multiplexing with fine-tuned gold and silver nanoshells, Chem. Sci. 8, 3038 (2017).

[52] P. B. Johnson and R. W. Christy, Optical constants of the noble metals, Phys. Rev. B 6, 4370 (1972).

[53] McGehee Group, Transfer matrix optical modeling, http://web. stanford.edu/group/mcgehee/transfermatrix/.

[54] G. V. Hartland, Optical studies of dynamics in noble metal nanostructures, Chem. Rev. (Washington, DC, US) 111, 3858 (2011).

[55] U. Kreibig, Small silver particles in photosensitive glass: Their nucleation and growth, Appl. Phys. 10, 255 (1976). 